\title{
RISK-BASED INVESTING IN THE GERMAN STOCK MARKET
}

\section{Jan Bastin*}

\begin{abstract}
The article shows properties of risk-based portfolios in the German stock market. Those systematic strategies use different approaches to weight stocks in portfolios. We present theoretical and empirical characteristics of five risk-based equity investments: the equal-weighted, minimum variance, maximum diversification and risk parity (equal risk budgeting and equal risk contribution) portfolios. Risk-based portfolios outperformed the market-cap weighted CDAX index with a lower level of risk in the period 2002-2015. Their excess returns relative to the CDAX index can be explained with Scherer's five-factor model; with Fama-French and low-risk anomaly factors. $\mathrm{R}^{2} \mathrm{~s}$ of different strategies range from $77 \%$ to $92 \%$.
\end{abstract}

Keywords: risk-based portfolio, German stock market, CDAX index, risk, returns, multifactor model

JEL Classification: G11, G10

\section{Introduction}

In the last decade, academic papers have documented an interesting behaviour of stock prices - the low-risk anomaly. In general, low-risk stocks tend to outperform high risk investments. This fact simply contradicts the core of finance; the risk-return trade-off. After the theoretical introduction of the Capital Asset Pricing Model (CAPM), Fama and MacBeth (1973) did not find a strong empirical relation between risk and return. The prediction of the CAPM seemed to be weak. Haugen and Baker (1991) demonstrated the performance of a portfolio with minimized variance of returns and reported comparable returns and a lower level of risk vis-à-vis a market-cap weighted index. Their text does not primary focus on the analysis of risks and returns, but the conclusion indicates that 1) a market-cap weighted portfolio is an inefficient investment; 2) characteristics of a minimum variance portfolio can be related to the low-risk anomaly (a perverse relation between risk and return). Later, Blitz and van Vliet (2007) showed that low-volatility stocks in U.S., Europe and Japan equity markets outperformed the market proxy as well as high-risk stocks in the period 1986-2006. Baker, Bradley and Wurgler (2011) formed quintiles by raking stocks by historical volatility or betas. The lowest risk measure groups were the most profitable in the period 1968-2008 in the U.S. market. Ang et al. (2006) and Ang et al. (2009) ranked stocks by residual volatility and demonstrated similar results as previous papers. Haugen

* Jan Bastin, Department of Banking and Insurance, University of Economics in Prague, Prague, Czech Republic (xbasj08@vse.cz);

This paper was created with the support of the Internal Grant Agency No. IG102024 "The Behaviour of Prices of Investment and Credit Instruments". 
and Baker (2012) claimed that the volatility effect is probably present in all observable equity markets in the world (in 33 different markets).

Classical financial models are not able to explain the low-risk anomaly. Thus professionals and scholars tried to find an alternative explanation. Blitz and van Vliet (2007) thought that the anomaly can exist because of leverage and administrative restrictions. Financial institutions are usually strongly regulated and executions of leverage and short-sale transactions are limited. Ang et al. (2009) proposed an explanation related to the analytical coverage and the ownership of institutions. Equity analysts tend to cover stocks with large institutional ownership. Those shares become more famous and thus more volatile. Prices of stocks with low institutional ownership tend to absorb new information more slowly. Haugen and Baker (2012) showed that asset management companies are not motivated to invest in low volatility stocks because of the investment process. Analysts tend to recommend popular stocks to their portfolio managers when planning the strategic allocation. This fact is better for both portfolio managers and clients because they would probably be satisfied to have famous firms in their portfolios. This results in overpricing of famous stocks. Authors conclude that asset management firms tend to invest in high risk stocks, analysts cover more volatile equities and financial media prefer to inform about high-volatility stocks. Baker, Bradley and Wurgler (2011) discussed two general reasons of the existence of the anomaly. The first one is related to the possibility of an irrational behaviour of investors explained by behavioural biases. The second one is related to the fact that investment firms tend to manage their portfolios relative to benchmarks. The primary objective of a portfolio manager is to maximize the information ratio (minimize the tracking error) instead of maximize the return-risk profile of the portfolio.

Our article presents equity low-risk strategies based on different ways to weight assets in portfolios. If the low-risk anomaly is able to persist in the long run, risk-based portfolios should be an interesting investment alternative. We demonstrate equal-weighted minimum variance, maximum diversification and risk parity portfolios and their empirical characteristics. Those strategies propose a significant advantage: we do not need to forecast returns of securities. We are interested only in risk measures. The objective of the paper is to explain and report empirical parameters of risk-based portfolios in the German Stock market. The text can also be considered as a free extension of Bastin (2016) who presented historical characteristics of minimum variance portfolios in Germany. We use the same data and the same period of the back-test.

We first explain principles of different risk-based strategies. Then, we describe data and methodologies. The results are demonstrated in the third part of the article; and finally we conclude the paper.

\section{Properties of Risk-Based Portfolios}

Below in the text we compare our investments to the CDAX index, an all-share market-cap weighted portfolio. Weights of those investments are calculated with market-caps of stocks. In general: 


$$
w_{i}=\frac{M C_{i}}{\sum_{i=1}^{N} M C_{i}},
$$

$w_{i}$ is the weight of security $i, M C_{i}$ is its market capitalization and $N$ is the number of companies in the universe. Most of worldwide equity indices are weighted by market-cap. This kind of portfolio is efficient in the risk-return world only if a set of assumptions is satisfied (see Haugen and Baker, 1991); all investors agree about the risk and the expected return for all securities; all investors can short-sell all securities; there are no taxes on security returns; an investment opportunity set for all investors holding any security in the index is restricted to the securities in the market-cap portfolio.

A different and simple way to allocate stock weights is the equal-weighted approach (EWP). An agent invests the same amount of money in each stocks of the universe.

$$
w_{i}=\frac{1}{N} \text {. }
$$

It is the reason why the EWP is often called "the 1/N strategy". We consider this method as a naive diversification. De Miguel, Garlappi and Uppal (2009) concluded that a portfolio with an equal-weighted allocation was the best performer between 14 different strategies in the Sharpe ratio context. The equal-weighted portfolio is efficient if all equities have the same returns, volatilities and correlations.

One has to launch an optimization procedure to obtain weights of the minimumvariance portfolio (MVP):

$$
\sigma_{p}^{2}=\sum_{i=1}^{N} \sum_{j=1}^{N} \sigma_{i, j} w_{i} w_{j} \rightarrow \text { minimize },
$$

where $\sigma_{p}^{2}$ is the variance of the portfolio $p$ and $\sigma_{i, j}$ is the covariance between returns of asset $i$ and asset $j$. We try to find the portfolio with the lowest variance of returns, theoretically defined by Markowitz (1952). Probably the first who reported the performance of minimumvariance portfolios were Haugen and Baker (1991). Their portfolios were created from a population of 1,000 of the largest equities in the U.S. They concluded that the minimum variance portfolio had lower risk and same/higher return than the market-cap weighted portfolio (Wilshire, 5,000). Clarke, de Silva and Thorley (2006) improved the previous work by a longer period studied (1968-2005) and other methods of covariance matrix estimation. Their results are similar to Haugen and Baker. A minimum variance portfolio is efficient by its definition. If all securities have the same expected returns, the MVP is the portfolio which maximizes the Sharpe ratio. We assume these fixed constraints in our analysis:

1. The maximum weight of an individual security in the portfolio is $5 \%$.

2. The sum of weights of all stocks in the portfolio is $100 \%$.

3. The maximum weight of an industry in the portfolio is $20 \%$.

4. The minimum weight of an individual security is $0 \%$.

Constraints ensure a sufficient level of diversification (the maximum asset and industry weight). Our portfolios are long-only and fully invested in equities. 
The maximum diversification approach (MDP) was introduced by Choueifaty and Coignard (2008). The objective function is the maximization of the diversification ratio:

$$
D R_{p=} \frac{\sum_{i=1}^{N} w_{i} \sigma_{i}}{\sigma_{p}}=\frac{\sum_{i=1}^{N} w_{i} \sigma_{i}}{\sum_{i=1}^{N} \sum_{j=1}^{N} \sigma_{i, j} w_{i} w_{j}} \rightarrow \text { maximize }
$$

$D R_{p}$ is the diversification ratio of portfolio $p$ and $\sigma_{i}$ is the volatility of security $i$. If securities are not perfectly correlated, the ratio will always be higher than 1 . The portfolio risk is smaller than the weighted average of stocks volatilities. A higher ratio means a greater diversification. Thus, we maximize the diversification ratio. If all stocks in the universe have the same volatility, the maximum diversification portfolio is equal to the minimum variance portfolio. Under certain conditions, we consider the maximum diversification portfolio to be an investment that maximizes its Sharpe ratio (if all stocks have the same Sharpe ratio). In the MDP case, we run optimization procedures with the same constraints used for the MVP.

The real application of the risk parity concept can be provided in various ways, but the theoretical idea is simple. Each asset in the portfolio should have the same contribution of risk to the total risk of the portfolio. We provide two calculations of risk parity weights in our text. We call them similarly as pointed out in Carvalho, Lu and Moulin (2012): Equal Risk Budgeting (ERB) and Equal Risk Contribution (ERC). The ERB portfolio weight is calculated:

$$
w_{i}=\frac{\frac{1}{\sigma_{i}}}{\sum_{i=1}^{N} \frac{1}{\sigma_{i}}} .
$$

Risk is equally divided between all stocks in the universe. High-risk stocks have small weights and low-risk stocks are overweighted. If all stocks have the same volatility, they will also have the same weight and the investment will be the EWP. Equities in the ERB portfolio contribute with the same risk to the total risk only if we have equal correlations for every pair of stocks. In reality, this is not satisfied. Thus, we apply the ERC strategy which takes correlations into account (see Maillard, Roncalli and Teiletche, 2010). The contribution to risk is defined as the product of a weight and marginal risk of an asset. The portfolio risk can be rewrite as:

$$
\sigma_{p}^{2}=\sum_{i=1}^{N} \sigma_{i}^{2} w_{i}^{2}+\sum_{i=1}^{N} \sum_{i<>j}^{N} \sigma_{i, j} w_{i} w_{j}
$$

Then, the marginal risk contribution (MRC) is:

$$
M R C_{i}=\frac{\partial \sigma_{p}^{2}}{\partial w_{i}}=\frac{w_{i} \sigma_{i}^{2}+\sum_{i<>j}^{N} \sigma_{i, j} w_{j}}{\sigma_{p}^{2}}
$$

The total risk contribution (TRC) is the product of the weight of the $i$-th asset and its marginal risk: 


$$
T R C_{i}=w_{i} M R C_{i}
$$

And the total risk of the portfolio can be evaluated as the sum of total risk contributions. Maillard, Roncalli and Teiletche (2010) showed that if we introduce a Beta estimation of the asset $i$, the weight of asset $i$ in the ERC portfolio is:

$$
w_{i}=\frac{\beta_{i}^{-1}}{\sum_{i=1}^{N} \beta_{i}^{-1}} .
$$

Unfortunately, the equation cannot be solved due to the endogenity of the calculation. " $w_{i}$ is a function of the component beta $\beta_{i}$ which by definition depends on the portfolio". Authors proposed two possible optimization problems. The first one minimizes the sum of squares of differences of risk contributions of any two stocks:

$$
\sum_{i=1}^{N} \sum_{j=1}^{N}\left(w_{i}\left[\sum w\right]_{i}-w_{j}\left[\sum w\right]_{j}\right)^{2}
$$

The second one is similar to the minimum variance of returns optimization procedure.

If an investor form an ERC equity portfolio with a low number of stocks, it is possible to use the first option or the minimum variance optimization with two additional constraints: the equality of all total risk contributions and the condition that the sum of total risk contributions is equal to the portfolio risk. In a case of a large set of securities, Clarke, de Silva and Thorley (2013) proposed an analytic solution to form a risk parity portfolio.

We apply an alternative equation proposed by Carvalho, Lu and Moulin (2012), who derived another optimization problem:

$$
\frac{\sqrt{\sum_{i=1}^{N} \sum_{j=1}^{N} \sigma_{i, j} w_{i} w_{j}}}{\left(\prod_{i=1}^{N} w_{i}\right)^{1 / N}} \rightarrow \text { minimization } .
$$

The equation shows that the ERC portfolio should have similar characteristics as MVP (minimization of the portfolio volatility) and EWP (maximization of the denominator). Because of potential $0 \%$ weights in portfolios, we modify the denominator in this form:

$$
\left(\prod_{i=1}^{N}\left[1+w_{i}\right]\right)^{1 / N}-1
$$

Note that our ERC portfolio is only an approximation of the general ERC approach the condition of equal total risk contributions of individual assets is not satisfied. We also assume "minimum variance portfolio" constraints but have a different objective function.

Thus, we demonstrate empirical characteristics of the CDAX index, EWP, MVP, MDP, ERB, ERC. Weights of stocks in EWP (Equation 2) and ERB (Equation 5) strategies are easily calculated. We have to run optimization procedures to define weights in MVP (Equation 3), MDP (Equation 4) and ERC (Equation 11) and we assume set constraints. All portfolios presented below are long-only and fully invested in stocks. Our investments depend only on risk measures and do not need to forecast equity returns. Choueifaty and Coignard (2008); Carvalho, Lu and Moulin (2012) and Clarke, de Silva and Thorley 
(2013) presented historical performances of various risk-based portfolios and reported their ability to outperform a market-cap weighted portfolio. Differently, they apply the low-risk strategies using U.S., European and Japanese stocks. Below, we demonstrate risk-based portfolios in the German stock market.

Carvalho, Lu and Moulin (2012) also tried to explain the variation of differences between returns of the low-risk portfolios and the market-cap index with a five factor regression. They used Fama-French factors (market, value and size) and additional factors related to risk (Beta and residual volatility). They showed that (for U.S., European and Japanese stocks) all factors were statistically significant in regressions of the EWP, ERB, ERC and MDP (long only). Only market, beta and residual volatility factors were statistically significant in the case of the MVP. Authors also claimed that the EWP was mainly explained by the exposure to small-cap equities. It was true also for ERB and ERC strategies. MVP and MDP were strongly exposed to low beta stocks. Those regression results are consistent with theoretical expectations. In comparison with the market-cap weighted portfolio, the EWP will, by its definition, overweight small-cap stocks and underweight shares with high market cap. Risk parity portfolios should have characteristics of both EWP and MVP; it means an exposure to small-cap stocks and low-risk stocks. Maillard, Roncalli and Teiletche (2010) showed that their ex-ante risk measures are higher than MVP ones but smaller than those of EWP. And finally, MVP and MDP should be significantly exposed to low-risk stocks (see Scherer, 2010). Inspired by these results, we also apply a similar model.

\section{Data and Methodology}

As mentioned above, the text is a free extension of Bastin (2016) who presented results of the minimum variance and equal-weighted strategies. Due to the same data set and methodology, we report the same figures concerning MVP and EWP. Nevertheless, we provide a brief recap of our data and methodology.

Our source of data is Bloomberg. We use members of the CDAX index (an all-share market-cap weighted index) in the period from March 2002 to March 2015. The main source of calculations is monthly total returns. We demonstrate characteristics of portfolios formed by the top 200 equities sorted by market capitalization. A EURIBOR 1M deposit is the risk-free rate proxy.

As of the end of each quarter in the analysed period, risk-based portfolios are created. A security is selected in portfolios if some conditions are satisfied (it is a common stock; it is a CDAX member as of the formation date; it has a return history of 60 months; it is present in the top 200 sorted by market-cap as of the formation data). We estimate covariance matrices and historical standard deviations with 60 months of historical data. When variables for weight definitions are calculated, we form risk-based portfolios (described in the text above). Weights of EWP and ERB are directly calculated - see Equation 2 and Equation 5. In our other cases we have to run optimization procedures to define weights of assets in portfolios. We reform our portfolios as of the end of each quarter. Finally we measure their returns. We substitute empty return fields of a stock by the average returns of the industry. The security came from until the next formation date (ends of quotation on the exchange) in cases that 
a stock disappears from the sample. It means that we sort stocks in 10 industries and calculate their average returns each month. We substitute returns of the energy industry by Utilities industry returns because of a low number of stocks.

We present risk-return characteristics of risk-based portfolios and the CDAX index; average returns, risk measures, risk-adjusted figures and others. The estimation of CAPM Alphas and Betas are provided via a classical regression model.

$$
R_{p, t}-R_{f, t}=\alpha_{p}+\beta_{p}\left(R_{C D A X, t}-R_{f, t}\right)+u_{p, t},
$$

$R_{p, t}$ is the portfolio yield in time $t, R_{f, t}$ is the risk-free rate proxy (EURIBOR 1M Deposit) in time $t, R_{C D A X, t}$ is the return of the CDAX index, $\alpha$ and $\beta$ are parameters to be estimated and $u_{p, t}$ are residuals.

\subsection{The Multifactor regression}

We run a five-factor regression model proposed by Scherer (2010) to explain the variation of excess returns of risk-based portfolio over the market-cap weighted CDAX index.

$$
\begin{aligned}
R_{p, t} & -R_{C D A X, t}=\alpha_{5 F}+\beta_{C D A X}\left(R_{C D A X, t}-R_{f, t}\right)+\beta_{S M B} R_{S M B}+\beta_{H M L} R_{H M L}+ \\
& +\beta_{L B M H B} R_{L B M H B}+\beta_{L R V M H R V} R_{L R V M H R V}+u_{p, t}
\end{aligned}
$$

$R_{S M B}$ is the difference between returns on equities with low market-cap and stocks with high market-cap, $R_{H M L}$ is the difference between returns on stocks with high book-to-market ratios and stocks with low ratios ${ }^{1}, R_{L B M H B}$ is the difference between returns of low-beta stocks and high beta stocks and $R_{\text {LRVMHRV }}$ is the difference between returns of stocks with low-residual variance of returns and stocks with high residual variance of returns. $\alpha_{5 F}$, $\beta_{C D A X}, \beta_{S M B}, \beta_{H M L}, \beta_{L B M H B}$ and $\beta_{L R V M H R V}$ are parameters to be estimated. One can see, that the equation is an extension of the Fama-French three factor model. There are two additional factors based on the low-risk anomaly.

Explanatory variables are returns of long-short portfolios. They are constructed in the same way as our risk-based portfolios (we use the same data). Factor portfolios are equalweighted and rebalanced on quarterly basis.

We calculate differences of returns of the CDAX index and a 1M EURIBOR deposit to form the market excess return over the risk-free rate. The formation of SMB and HML is provided in a similar way as pointed out in Davis, Fama and French (2000). As of the end of each quarter we rank stocks by market-cap and allocate them in 3 groups. Small stocks (S) are bellow the 30th percentile of market-caps for stocks in our sample. Big stocks (B) are in the top 30 percent and mid-cap stocks are allocated in the middle group (M). In every group we then rank stocks by book-value-price ratio (BV/P) and divide them in another 3 groups: $\mathrm{H}$ (the highest 30 percent of $\mathrm{BV} / \mathrm{P}$ ), $\mathrm{MM}$ (the middle 40 percent) and $\mathrm{L}$ (the lowest

1 We substitute the book-to-market ratio by the "book value per share - price" ratio. We use a 6-month lag of the book value per share (as of time $t-6$ ) and the last price (as of time $t$ ) to calculate the ratio. This time gap should ensure that book values per share were publicly available as of formation dates of portfolios. 
30 percent). Thus we create 9 portfolios in which our stock universe is selected; $\mathrm{S} / \mathrm{L}, \mathrm{S} / \mathrm{MM}$, $\mathrm{S} / \mathrm{H}, \mathrm{M} / \mathrm{L}, \mathrm{M} / \mathrm{MM}, \mathrm{M} / \mathrm{H}, \mathrm{B} / \mathrm{L}, \mathrm{B} / \mathrm{MM}$ and B/H. The $\mathrm{S} / \mathrm{L}$ group contains smallest stocks with lowest $\mathrm{BV} / \mathrm{P}$ ratio, the $\mathrm{S} / \mathrm{H}$ group invests in small stocks with highest $\mathrm{BV} / \mathrm{P}$ ratio, etc. Long-short returns of the "Small minus Big" portfolio are:

$$
R_{S M B}=\frac{\left(R_{S / L}+R_{S / M M}+R_{S / H}\right)}{3}-\frac{\left(R_{B / L}+R_{B / M M}+R_{B / H}\right)}{3} .
$$

Thus the RSMB factor is the difference between averages of returns on small-equity groups and big-stocks groups. Returns on the "High minus Low" portfolio are calculated similarly; it is the difference between returns on high BV/P portfolios and low BV/P groups:

$$
R_{H M L}=\frac{\left(R_{S / H}+R_{M / H}+R_{B / H}\right)}{3}-\frac{\left(R_{S / L}+R_{M / L}+R_{B / L}\right)}{3} .
$$

We note that as of the beginning of our testing period both $R_{S M B}$ and $R_{H M L}$ are biased. In the years 2002, 2003 and 2004, on average, 25\% of stocks (in our sample) have empty fields of book values data as of ends of quarters. There are also missing data of book values in other years, but the percentage of missing data is very low (on average below $4 \%$ as of ends of quarters). Shmueli (2010) discussed the problem of missing values in the context of explanation and prediction. In general, if a small percentage of missing data appears in the sample, one can throw out missing values in cases of estimation. On the other hand, this is not possible in the prediction context. For the purpose of our multifactor model ${ }^{2}$, we decide to exclude stocks with missing book values from the potential allocation to SMB and HMB groups as of formation dates. Nevertheless, we believe that our SMB and HML groups are good proxies of real ones ${ }^{3}$.

We have to estimate betas and residual variances of each stock in our sample to calculate returns of LBMHB ("Low beta minus High beta") and LRVMHRV ("Low residual variance minus High residual variance") portfolios. Betas and residual variances are estimated with the Equation 13, applied for individual stocks, with 60 months of historical data. The 1-month EURIBOR rate is available from the end of 1998 in our sample. Thus we used a 1-month FIBOR deposit yield as the risk-free rate proxy before 31 December 1998.

Then, we allocate stocks in LBMHB and LRVMHRV in a similar way as described for the SMB and HML. We rank stocks by residual variance in 3 groups (the lowest 30\%, 40\% and the highest $30 \%$ ). We then provide the beta ranking procedure in each residual variance groups. Thus we allocate equities in 9 "risk-factor" portfolios similarly to the approach used for the SMB and HML formation. We shortly introduced the low-risk anomaly above in the text. It means that returns of risk-factor long-short portfolios should be positive.

2 We do not provide predictions; our objective is to explain.

3 We construct an alternative SMB portfolio (not shown) only by selecting equities by market-cap in 3 groups of 60, 80 and 60 stocks (with no missing values; all market-cap data are available). The correlation between biased and no-missing stocks SMB returns is $98 \%$. We also calculate correlations of all pairs of factors with no-missing stocks SMB and figures are very similar to that with biased numbers. 
As described in Scherer (2010) and Carvalho, Lu and Moulin (2012) we also adjust long-short returns time series with a hedge ratio:

$$
R_{L B M H B}=\left(R_{L B}-R_{f}\right)-\frac{\beta_{L B}}{\beta_{H B}}\left(R_{H B}-R_{f}\right) .
$$

where $R_{L B}$ is the return of the lowest beta equites, $R_{H B}$ is the return of the highest beta stocks. $\beta_{L B}$ and $\beta_{H B}$ are their ex-ante betas. This leads to a beta neutral time series. The same procedure is used to form the LRVMHRV portfolio.

\section{Empirical Results}

We demonstrate historical characteristics of low-risk portfolios and the CDAX index in Table 1. All risk-based portfolios generated the higher rates of returns than the marketcap weighted CDAX index. The ERB portfolio was the best performer in terms of average returns (12.2\% annualized). The MVP had similar average returns in comparison with the CDAX. On the other hand, investors are more interested in geometric returns in reality; and the MVP outperformed in this purpose. As anticipated, our portfolios had lower risk measures (both standard deviations and betas) than the CDAX. MVP had the lowest standard deviation figure (8.5\% annualized), whereas the lowest CAPM beta was produced by the ERC portfolio (0.25). Thus, all portfolios demonstrated a significant reduction of ex-post risk figures. This reduction is more visible in case of optimization-based strategies (MVP, MDP and ERC). Their volatility was more than about 50\% lower than the standard deviation of the index and their Betas are lower than 0.3. In terms of risk-adjusted performance, the best figures were produced by the ERC portfolio with a Sharpe ratio of 0.32 and an annualized CAPM Alpha of 7.5\%. Risk-based portfolios had all higher Sharpe ratios than the CDAX and also positive and statistically significant CAPM Alphas. Our results show that the CDAX index was an inefficient investment opportunity in the period 2002-2015.

As anticipated, the MVP has the lowest standard deviation measure. The MDP has both higher standard deviation and returns than the MVP. In the context of theoretical risk-return expectations, the interpretation of ERC figures is harder. It should have risk and return figures between those of MVP and EWP. Our empirical results show that the ERC has comparable risks figure to that produced by the MVP and also comparable returns to the EWP.

We plot portfolios in the risk-return space (see Figure 1). One can see a perverse trade-off between risk and return, a result potentially related to the low-risk anomaly (see Ang et al., 2006). The CDAX index is a dominated investment opportunity in comparison with risk-based portfolios. As a riskier investment, it is more affected by bear markets. And our analysed period (2002-2015) contains two huge drawdowns. Thus, it should not be surprising that the index performed worse.

We measured year-by-year returns from the end of March of year Y to the end of March of year $\mathrm{Y}+1$ in Table 2. Portfolios formed by an optimization procedure (MVP, MDP and ERC) had the smallest drawdowns in bear market years. The MVP and ERC had only 2 years of negative 1-year returns. 
Table 1 | Empirical Characteristics of Risk-Based Portfolios in the Period 2002-2015

\begin{tabular}{|l|c|c|c|c|c|c|}
\hline & CDAX & EWP & MVP & MDP & ERB & ERC \\
\hline Average Returns (\%) & 0.76 & 1.00 & 0.69 & 0.81 & 1.02 & 0.93 \\
\hline Geometric Average Returns (\%) & 0.57 & 0.90 & 0.66 & 0.78 & 0.94 & 0.90 \\
\hline Standard Deviation (\%) & 5.98 & 4.39 & 2.46 & 2.72 & 3.86 & 2.47 \\
\hline Beta (CDAX) & 1.00 & 0.66 & 0.29 & 0.27 & 0.57 & 0.25 \\
\hline Sharpe ratio & 0.10 & 0.19 & 0.22 & 0.24 & 0.22 & 0.32 \\
\hline Alpha (CDAX) (\%) & - & 0.45 & 0.37 & 0.50 & 0.52 & 0.63 \\
\hline t(Alpha) & - & $2.82^{* * *}$ & $2.54^{* *}$ & $2.78^{* * *}$ & $3.56^{* *}$ & $3.94^{* * *}$ \\
\hline Cumulative Returns (\%) & 144.20 & 303.79 & 180.57 & 234.24 & 330.56 & 306.15 \\
\hline
\end{tabular}

Note: $t$ (Alpha) is the $t$-statistics $\left(*^{* *}, * *{ }^{*}\right.$ denote rejection of the null at the $1 \%, 5 \%$ and $10 \%$ level of significance) Source: Own analysis

Figure 1 | Risk-Based Portfolios in the Risk-Return Space

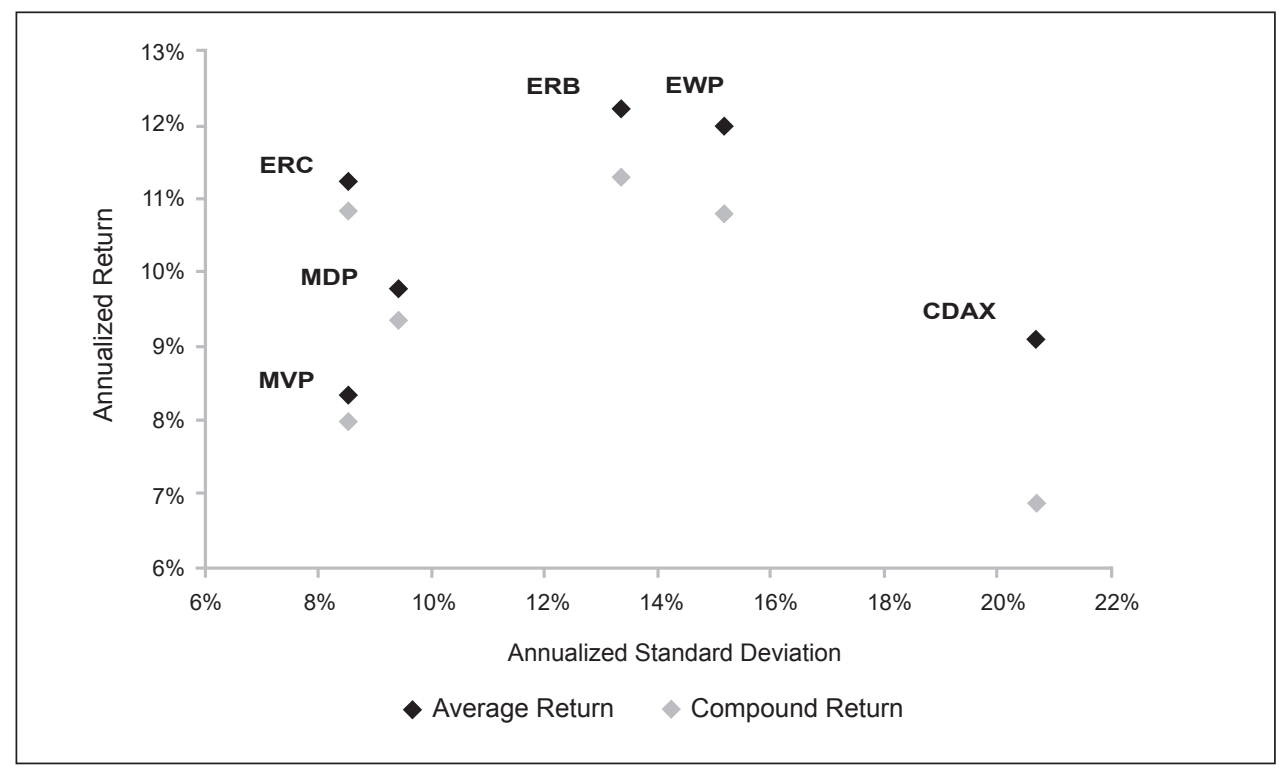

Source: Own analysis

It seems that risk-based portfolios are able to outperform the CDAX index in bear markets. On the other hand, in general, they are not able to outperform the index in bull markets. Thus, we present figures of our portfolios when the CDAX returns are positive (the bull market proxy) and negative (the bear market proxy) in Tables 3 and 4. Those tables confirm the facts about abilities of risk-based portfolios outperformance in bull/bear markets. 
Table 2 | Year-by-Year Returns of Risk-Based Portfolios

\begin{tabular}{|l|c|c|c|c|c|c|}
\hline Year & CDAX (\%) & EWP (\%) & MVP (\%) & MDP (\%) & ERB (\%) & ERC (\%) \\
\hline $\mathbf{2 0 0 2}$ & -51.13 & -22.42 & 0.04 & -6.25 & -16.12 & 4.82 \\
\hline $\mathbf{2 0 0 3}$ & 59.15 & 55.74 & 19.56 & 19.68 & 48.14 & 22.76 \\
\hline $\mathbf{2 0 0 4}$ & 13.21 & 17.87 & 14.89 & 18.48 & 18.44 & 22.07 \\
\hline $\mathbf{2 0 0 5}$ & 39.76 & 41.29 & 24.60 & 29.90 & 37.82 & 30.35 \\
\hline $\mathbf{2 0 0 6}$ & 17.37 & 22.00 & 10.98 & 19.78 & 21.66 & 19.89 \\
\hline $\mathbf{2 0 0 7}$ & -7.09 & -13.48 & -9.03 & -3.68 & -11.08 & -5.86 \\
\hline $\mathbf{2 0 0 8}$ & -40.57 & -37.81 & -18.28 & -17.89 & -33.54 & -15.19 \\
\hline $\mathbf{2 0 0 9}$ & 53.49 & 59.87 & 12.13 & 20.25 & 55.35 & 18.41 \\
\hline $\mathbf{2 0 1 0}$ & 16.30 & 26.37 & 16.34 & 15.00 & 24.36 & 19.74 \\
\hline $\mathbf{2 0 1 1}$ & -1.74 & -4.30 & 4.08 & -7.19 & -1.77 & 1.11 \\
\hline $\mathbf{2 0 1 2}$ & 13.66 & 12.48 & 14.57 & 16.56 & 13.29 & 13.15 \\
\hline $\mathbf{2 0 1 3}$ & 23.09 & 22.34 & 12.33 & 18.97 & 18.94 & 9.69 \\
\hline $\mathbf{2 0 1 4}$ & 24.77 & 14.91 & 13.66 & 14.82 & 15.46 & 16.46 \\
\hline Average & 12.33 & 14.99 & 8.91 & 10.65 & 14.69 & 12.11 \\
\hline Standard Deviation & 30.83 & 27.71 & 11.42 & 13.74 & 24.33 & 12.32 \\
\hline
\end{tabular}

Source: Own analysis

Table 3 | Empirical Characteristics of Risk-Based Portfolios in Bull Markets

\begin{tabular}{|l|c|c|c|c|c|c|}
\hline \multicolumn{1}{|c|}{ BULL MARKET } & CDAX & EWP & MVP & MDP & ERB & ERC \\
\hline Average Returns (\%) & 4.33 & 3.48 & 1.82 & 1.85 & 3.18 & 1.91 \\
\hline $\begin{array}{l}\text { Geometric Average } \\
\text { Returns (\%) }\end{array}$ & 4.27 & 3.45 & 1.81 & 1.83 & 3.15 & 1.89 \\
\hline Standard Deviation (\%) & 3.41 & 2.59 & 1.75 & 2.08 & 2.24 & 1.94 \\
\hline Beta (CDAX) & 1.00 & 0.57 & 0.20 & 0.16 & 0.49 & 0.15 \\
\hline Sharpe ratio & 1.23 & 1.29 & 0.96 & 0.83 & 1.36 & 0.91 \\
\hline
\end{tabular}

Source: Own analysis

Table 4 | Empirical Characteristics of Risk-Based Portfolios in Bear Markets

\begin{tabular}{|l|r|r|r|r|r|r|}
\hline \multicolumn{1}{|c|}{ BEAR MARKET } & CDAX & EWP & MVP & MDP & ERB & ERC \\
\hline Average Returns (\%) & -4.65 & -2.77 & -1.02 & -0.76 & -2.26 & -0.54 \\
\hline $\begin{array}{l}\text { Geometric Average } \\
\text { Returns (\%) }\end{array}$ & -4.79 & -2.85 & -1.05 & -0.80 & -2.32 & -0.58 \\
\hline Standard Deviation (\%) & 4.86 & 3.83 & 2.40 & 2.83 & 3.48 & 2.45 \\
\hline Beta (CDAX) & 1.00 & 0.64 & 0.29 & 0.30 & 0.57 & 0.29 \\
\hline Sharpe ratio & -0.99 & -0.77 & -0.50 & -0.33 & -0.70 & -0.29 \\
\hline
\end{tabular}

Source: Own analysis 
Additionally we plot cumulative returns of low-risk portfolios in Figure 3. EWP, ERB and ERC have similar returns (the highest are produced by ERB). Risk-based portfolios are a good solution in high-volatility times. Their drawdowns in bad times are lower in comparison with the CDAX index.

Baker, Bradley and Wurgler (2011) argued that institutional investors manage their portfolios relative to benchmarks and they are interested in maximizing the information ratio. It can be one of reasons of the existence of the low-risk anomaly. We show portfolio-benchmark annualized figures in Table 5 and assume the CDAX to be the benchmark. MVP, MDP and ERC have the largest tracking errors and the lowest information ratios. In term of portfolio relative to benchmark comparison, these strategies are less attractive then EWP and ERB.

Figure 2 | Cumulative Returns of Portfolios in the Period 2002-2015

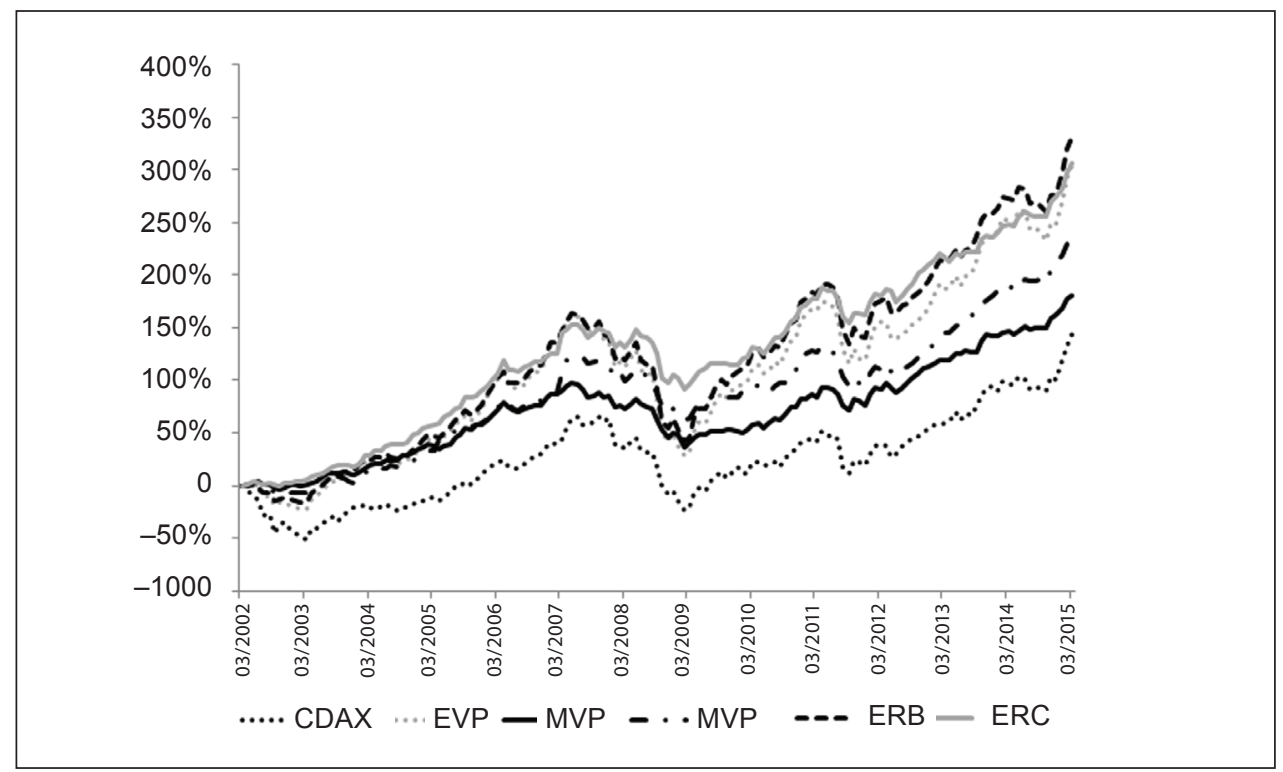

Source: Own analysis

Table 5 | Portfolio Relative to Benchmark Annualized Characteristics

\begin{tabular}{|l|r|r|r|r|r|}
\hline & EWP & MVP & MDP & ERB & ERC \\
\hline Outperformance (\%) & 2.86 & -0.77 & 0.67 & 3.10 & 2.10 \\
\hline Geometric outperformance (\%) & 3.90 & 1.07 & 2.43 & 4.40 & 3.9 \\
\hline Tracking Error (\%) & 9.78 & 16.00 & 16.97 & 10.79 & 16.90 \\
\hline IR & 0.29 & -0.05 & 0.04 & 0.29 & 0.12 \\
\hline Geometric IR & 0.40 & 0.07 & 0.14 & 0.41 & 0.23 \\
\hline
\end{tabular}

Source: Own analysis 
The next figure shows the number of equities in low-risk portfolios. We demonstrate only 4 portfolios. The EWP and ERB contain, by their definition, all stocks in our universe (200 securities). Due to the fact, that we do not apply turnover constraints, the number of stocks sometimes widely changes in analysed quarters, especially in the ERC case. This fact simply means higher transaction costs in reality. Transaction costs were not incorporated in our analysis.

Figure 3 | The Number of Stocks in Risk-Based Portfolios in the Period 2002-2015

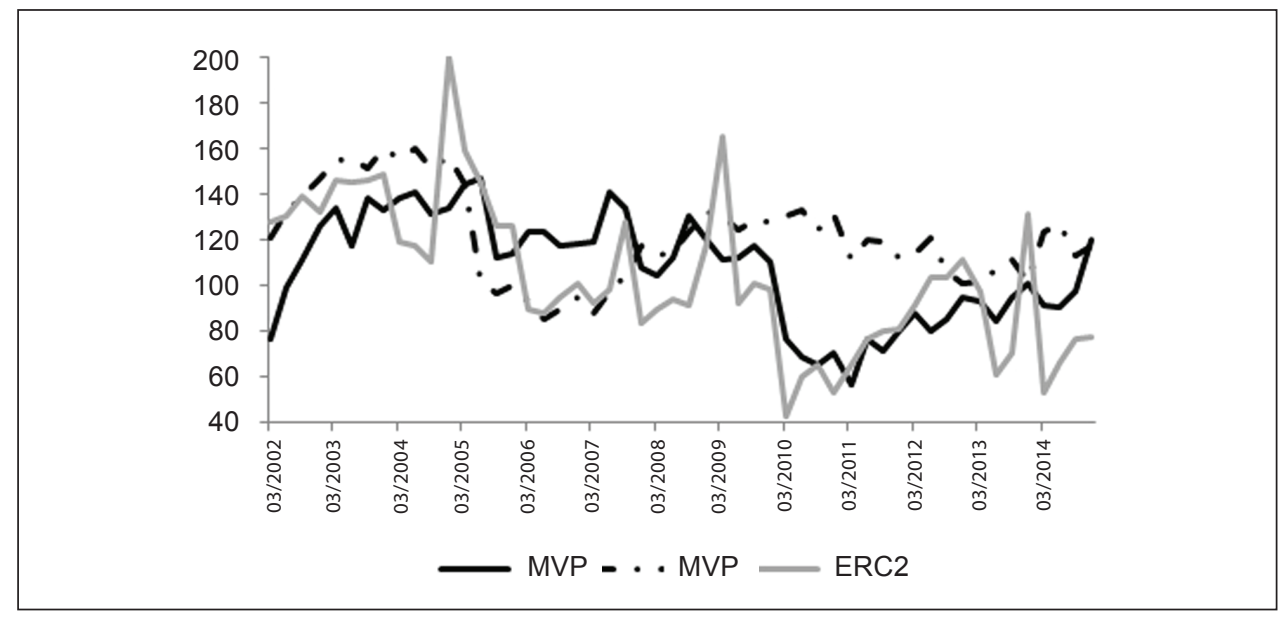

Source: Own analysis

We next demonstrate results of the multifactor regression model. They can be compared to that showed in Carvalho, Lu and Moulin (2012) who applied a similar model also in the regional Europe stock universe.

We show empirical characteristics of factors in Table 6. During our testing period, the annualized differential of the CDAX and the risk-free proxy was $7.3 \%$. The "size effect" was not present in our test; big stocks outperformed small stocks by $0.8 \%$ annually. High $\mathrm{BV} / \mathrm{P}$ ratio firms performed better than small BV/P companies (annualized difference: $1.9 \%$ ). As mentioned, low risk investments are probably a good choice in high volatility period. Annualized differentials of returns of risk portfolios are higher than $6 \%$.

The correlation matrix of factors is demonstrated in Table 7. HML returns and CDAX excess returns are correlated at $64 \%$. The negative correlation of CDAX excess and SMB returns arise from the fact, that CDAX is a market-cap weighted portfolio and thus big stocks have higher weights. Correlations between HML and SMB returns should be smaller due to their construction process. The same is true in LBMHB and LRVMHRV. It is interesting that correlations of risk factors and CDAX excess returns are zero.

Results of the multifactor regression are shown in Table 8. Null hypothesis are zero values of estimated parameters. All intercepts $\left(\alpha_{5 \mathrm{~F}}\right)$ of risk-based strategies are not significantly different from zero. Rs-squared are all high (from $77.3 \%$ to $92.6 \%$ ) and Durbin-Watson statistics are also all at a reasonable level. 
Table 6 | Empirical Characteristics of Factors in the Period 2002-2015

\begin{tabular}{|l|r|r|r|r|c|}
\hline & $\boldsymbol{R}_{\text {CDAX-Rf }}$ & $\boldsymbol{R}_{\text {SMB }}$ & $\boldsymbol{R}_{\text {HML }}$ & $\boldsymbol{R}_{\text {LBMHB }}$ & $\boldsymbol{R}_{\text {LRVMHRV }}$ \\
\hline Average (\%) & 0.61 & -0.07 & 0.16 & 0.52 & 0.58 \\
\hline Standard Deviation (\%) & 6.01 & 2.77 & 3.49 & 1.87 & 1.86 \\
\hline
\end{tabular}

Source: Own analysis

Table 7 | Correlations of Factors in the Period 2002-2015 (\%)

\begin{tabular}{|l|c|c|c|c|c|}
\hline & $\boldsymbol{R}_{\text {CDAX-Rf }}$ & $\boldsymbol{R}_{\text {SMB }}$ & $\boldsymbol{R}_{\text {HML }}$ & $\boldsymbol{R}_{\text {LBMHB }}$ & $\boldsymbol{R}_{\text {LRVMHRV }}$ \\
\hline $\boldsymbol{R}_{\text {CDAX-Rf }}$ & - & - & - & - & - \\
\hline $\boldsymbol{R}_{\text {SMB }}$ & -51.66 & - & - & - & - \\
\hline $\boldsymbol{R}_{\text {HML }}$ & 63.84 & -33.01 & - & - & - \\
\hline $\boldsymbol{R}_{\text {LBMHB }}$ & 0.00 & 33.18 & -35.18 & - & - \\
\hline $\boldsymbol{R}_{\text {LRVMHRV }}$ & 0.00 & -28.14 & -17.10 & 0.19 & - \\
\hline
\end{tabular}

Source: Own analysis

Excess returns of the EWP are mainly explained with four statistically significant factors. The EWP is exposed to small, high BV/P and low beta stocks. This finding should be correct because the EWP, by its definition, overweight small stocks and underweight big equities (in comparison with a market-cap weighted portfolio). The MVP is exposed to small, low beta and low residual variance stocks. The small stocks exposition contradicts Scherer's (2010) findings; on the other hand, parameters demonstrated by Carvalho, Lu and Moulin (2012) confirm our result. Only three factors are significant in the MDP case. It is significantly exposed to high BV/P and low beta stocks. All factors are statistically significant in regressions of ERB and ERC. Thus risk parity portfolios meet theoretical expectations (similar properties to EWP and MVP), while they are exposed to small stocks and low risk stocks.

All strategies excess returns relative to the index are negatively exposed to the CDAX excess returns and positively exposed to low beta stocks. This makes risk-based portfolios to be defensive investments. In general, our regression results are similar and comparable to that showed by Carvalho, Lu and Moulin (2012) and have a strong explanation power. We can recognize few differences in term of significance of parameters (for example LRVMHRV is insignificant in MDP case in our result).

To be correct, we note that some requirements of a regression model estimated with the ordinary least square method are not satisfied (residuals should not be auto-correlated, they should be normally distributed and homoscedastic). We apply Ljung-Box Q-statistics ${ }^{4}$ to test the presence of autocorrelation in residual terms, the $\mathrm{ARCH}$ test to identify heteroscedasticity and Jarque-Bera statistics to test the normality of residuals. Diagnostics of residuals are shown in Table 9.

$4 \quad$ We observe twelfth lags. 
Table 8 | Regression Coefficients of the Multifactor Model

\begin{tabular}{|l|c|c|c|c|c|}
\hline & EWP & MVP & MDP & ERB & ERC \\
\hline $\boldsymbol{a}_{5 F}$ & 0.0014 & 0.0002 & 0.0006 & 0.0013 & 0.0009 \\
\hline $\boldsymbol{t}\left(\boldsymbol{a}_{5 F}\right)$ & 1.1912 & 0.1748 & 0.3976 & 1.1388 & 0.7772 \\
\hline $\boldsymbol{\beta}_{\text {CDAX-Rf }}$ & -0.3293 & -0.6737 & -0.7836 & -0.4219 & -0.7639 \\
\hline $\boldsymbol{t}\left(\boldsymbol{\beta}_{\text {CDAX-Rf }}\right)$ & $-11.0965^{* * *}$ & $-21.6497^{* * *}$ & $-22.5663^{* * *}$ & $-15.6334^{* * *}$ & $-25.9909^{* * *}$ \\
\hline $\boldsymbol{\beta}_{\text {SMB }}$ & 0.4165 & 0.1591 & 0.0969 & 0.3659 & 0.2121 \\
\hline $\boldsymbol{t}\left(\boldsymbol{\beta}_{\text {SMB }}\right)$ & $7.6876^{* * *}$ & $2.7995^{* * *}$ & 1.5276 & $7.4241^{* * *}$ & $3.9524^{* * *}$ \\
\hline $\boldsymbol{\beta}_{\text {HML }}$ & 0.2389 & 0.0061 & 0.2120 & 0.2289 & 0.1911 \\
\hline $\boldsymbol{t}\left(\boldsymbol{\beta}_{\text {HMLL }}\right)$ & $4.9908^{* * *}$ & 0.1219 & $3.7856^{* * *}$ & $5.2570^{* * *}$ & $4.0313^{* * *}$ \\
\hline $\boldsymbol{\beta}_{\text {LBMHB }}$ & 0.4223 & 0.4762 & 0.8614 & 0.4355 & 0.6903 \\
\hline $\boldsymbol{t}\left(\boldsymbol{\beta}_{\text {LBMHB }}\right)$ & $5.7640^{* * *}$ & $6.1989^{* * *}$ & $10.0481^{* * *}$ & $6.5355^{* * *}$ & $9.5136^{* * *}$ \\
\hline $\boldsymbol{\beta}_{\text {LRVMHRV }}$ & 0.1128 & 0.1478 & -0.0004 & 0.2611 & 0.2953 \\
\hline $\boldsymbol{t}\left(\boldsymbol{\beta}_{\text {LRVMHRV }}\right)$ & $1.7552^{*}$ & $2.1931^{* *}$ & -0.0048 & $4.4668^{* * *}$ & $4.6376^{* * *}$ \\
\hline $\boldsymbol{R}^{2}$ & 0.7734 & 0.9070 & 0.8971 & 0.8461 & 0.9256 \\
\hline $\boldsymbol{D} W$ & 2.0250 & 2.1144 & 2.0348 & 2.0227 & 2.0727 \\
\hline
\end{tabular}

Note: $t(\beta)$ is the $t$-statistic $(* * *, * * *$ denote rejection of the null at the $1 \%, 5 \%$ and $10 \%$ level of significance) Source: Own analysis

Table 9 | Diagnostics of Residual Terms

\begin{tabular}{|l|c|c|c|c|c|}
\hline & EWP & MVP & MDP & ERB & ERC \\
\hline Autocorrelation & NO & NO & YES*** & YES* & YES** \\
\hline Heteroscedasticity & YES** & NO & NO & YES** & NO \\
\hline Jarque-Bera & 0.2427 & $5.2892^{*}$ & $7.491^{* *}$ & 0.4361 & $9.9772^{* * *}$ \\
\hline
\end{tabular}

Note: $* * * * * *$ denote rejection of the null at the $1 \%, 5 \%$ and $10 \%$ level of significance Source: Own analysis

Autocorrelation is not present in residuals of EWP and MVP models, but it is generally present in the rest of our models. ARCH tests also identify heteroscedasticity in EWP and ERB models. We reject the null hypothesis of normal distributions in cases of MDP and ERC residuals. Thus our estimates are not BLUE5 ${ }^{5}$. When applying both White and Newey-West heteroscedasticity standard errors adjustments (not showed), values of $t$-statistics decrease, but the majority of them remain statistically significant. 


\section{Conclusion}

We demonstrated empirical characteristics of portfolios formed by risk-based procedures in the German stock market; equal-weighted, minimum variance, maximum diversification and risk parity portfolios. These systematic strategies, probably related to the low-risk anomaly, were interesting for many researchers in last years.

The most efficient strategy, in our testing sample, is the Equal Risk Contribution portfolio. Its average returns are about $20 \%$ higher and its standard deviation is about $50 \%$ lower in comparison with measures of the market-cap weighted CDAX index.

In general, our text confirms findings of previously published papers. Our risk-based portfolios outperformed a market-cap weighted portfolio with a lower level of risk. Thus, they had better risk-adjusted performance results. On the other hand, risk-based investments were not able to outperform in bull markets. Our results can be affected by the period studied; two huge bear markets were present in the lost decade (2000-2010) - the "dot. com" drawdown and the financial crisis period. Naturally, low-risk investments perform better in high-volatility periods. Thus the outperformance of risk-based portfolios in our paper should not be surprising. One can argue, that if low risk investments underperform a market-cap weighted portfolio in bull markets, they would probably underperform in the very long run. We are not able to contradict this thesis, but cited papers do. They report similar results with longer periods studied.

Inspired by Carvalho, Lu and Moulin (2012), we showed figures of a multifactor model to explain the variation of risk-based portfolios returns relative to that of the CDAX and report similar results. In general, the CDAX excess return, size factor, value factor and factors related to beta and residual variance are doing a good job in this explanation. On the other hand, our models have problems in residual diagnostics.

We consider the application of risk-based strategies with large number of equities in the recent history of the German stock market development as the main contribution of the text. However, to be correct, we note that performances and characteristics of our portfolios were analysed only in the German stock market alone and a single period (due to historical data availability). Therefore we cannot generalize our results to all stock markets and all potential historical periods. On the other hand, articles cited above tested risk-based portfolios in U.S., Japan and Europe in different periods compared to our analysis. Their conclusions are similar to our findings. Thus, we believe that risk-based portfolios should have similar properties in other developed markets. In fact, we chose the German stock market because of its size (top 10 worldwide), advancement, geographical situation (as a European comparison to traditional applications in the U.S.) and large number of high-liquid stocks. In a regional/emerging markets/CEE context, the situation can be different because of lower number of high liquid stocks. Bastin (2015) studied performances of minimum variance portfolios in the Prague stock exchange, a small regional stock market. He concludes that MVPs outperformed the market-cap weighted PX index in the period 2006-2013, but provide a warning: "Investing on the Czech stock market alone is not a clever way to diversify risk". The period studied is too short and the number of stocks 
in MVPs ranged from 4 to 15 . Thus, a small regional market should be seen only as a part of an investor's equity portfolio.

Baker, Bradley and Wurgler (2011) argued that most of mutual funds use a popular index (which is usually market-cap weighted) as a benchmark to manage their portfolios. If we accept that a market-cap weighted portfolio is a dominated investment, financial institutions may ratiocinate about modifications in benchmark definitions or asset allocations. It seems that a market-cap weighted portfolio is a bad proxy solution for the real market portfolio (which is efficient by its definition). Risk-based portfolios are not market portfolio proxies, but the empirical evidence shows that they can be proposed as an interesting investment allocation alternative to the traditional market-cap investing.

\section{References}

Ang, A., Hodrick, R. J., Xing, Y., Zhang, X. (2006). The Cross-Section of Volatility and Expected Returns. The Journal of Finance, 61(1), 259-299.

(2009). High Idiosyncratic Volatility and Low Returns: International and Further U.S. Evidence. The Journal of Financial Economics, 91(1), 1-23.

Baker, M., Bradley, B., Wurgler, J. (2011). Benchmarks as Limits to Arbitrage: Understanding the Low-Volatility Anomaly. Financial Analysts Journal, 67(1), 40-54.

Baker, N. L., Haugen, R. A. (2012). Low Risk Stocks Outperform within All Observable Markets of the World. Social Science Research Network. [online] Available at: http://ssrn.com/ abstract=2055431. [Accessed: 13 June 2013], https://doi.org/10.2139/ssrn.2055431

Bastin, J. (2015). Minimum Variance Investing on the Prague Stock Exchange. 18th International Scientific Conference "Enterprise and the Competitive Environment". Brno: Mendel University, 2015, 46-55. [online] Available from: https://ece.pefka.mendelu.cz/sites/ default/files/imce/ece_2015_final.pdf. [Accessed: 2 November 2015]

(2016). Minimum Variance Portfolios in the German Stock Market. Prague Economic Papers, [online] available from: http://dx.doi.org/10.18267/j.pep.599.

Blitz, D. C., van Vliet, P. (2007). The Volatility Effect: Lower Risk without Lower Return. Journal of Portfolio Management, 34(1), 102-113.

Carvalho, R., Lu, X., Moulin, P. (2012). Demystifying Equity Risk-Based Strategies. Journal of Portfolio Management, 38(3), 56-70, https://doi.org/10.3905/.jpm.2012.38.3.056

Choueifaty, Y., Coignard, Y. (2008). Towards Maximum Diversification. Journal of Portfolio Management, 35(1), 40-51, https://doi.org/10.3905/jpm.2008.35.1.40

Clarke, R. G., De Silva, H., Thorley, S. (2006). Minimum Variance Portfolios in the U.S. Equity Market. Journal of Portfolio Management, 33(1), 10-24, https://doi.org/10.3905/jpm.2006.661366

(2013). Risk Parity, Maximum Diversification, and Minimum Variance: An Analytic Perspective. Journal of Portfolio Management, 39(3), 39-53,

https://doi.org/10.3905/jpm.2013.39.3.039

Davis, J. L., Fama, E. F., French, K. R. (2000). Characteristics, Covariances, and Average Returns: 1929 to 1997. The Journal of Finance, 55(1), 389-406, https://doi.org/10.1111/0022-1082.00209

DeMiguel, V., Garlappi, L., Uppal, R. (2007). Optimal versus Naive Diversification: How Inefficient is the 1/N Portfolio Strategy? The Review of Financial Studies, 22(5), 1915-1953, https://doi.org/10.1093/rfs/hhm075 
Fama, E. F., Mac Beth, J. D. (1973). Risk, Return and Equilibrium: Empirical Tests. Journal of Political Economy, 81(3), 607-636,https://doi.org/10.1086/260061

Haugen, R., Baker, N. (1991). The Efficient Market Inefficiency of Capitalization-Weighted Stock Portfolios. Journal of Portfolio Management, 17(3), 35-40, https://doi.org/10.3905/jpm.1991.409335

Maillard, S., Roncalli, T., Teiletche, J. (2010). The Properties of Equally Weighted Risk Contribution Portfolios. Journal of Portfolio Management, 36(4), 60-70, https://doi.org/10.3905/jpm.2010.36.4.060

Markowitz, H. (1952). Portfolio Selection. The Journal of Finance, 7(1), 77-91, https://doi.org/10.2307/2975974

Scherer, B. (2010). A New Look at Minimum Variance Investing. Social Science Research Network. [online] Available at: http://ssrn.com/abstract=1681306. [Accessed: 15 December 2014], https://doi.org/10.2139/ssrn.1681306

Shmueli, G. (2010). To Explain or to Predict? Statistical Science, 25(3), 289-310. 